\title{
Mycobacterium bovis BCG infection severely delays Trichuris muris expulsion and co-infection suppresses immune responsiveness to both pathogens
}

Hendrik J Nel ${ }^{1,2^{*}}$, Nelita du Plessis ${ }^{1 *}$, Leanie Kleynhans ${ }^{1}$, André G Loxton ${ }^{1}$, Paul D van Helden ${ }^{1}$ and Gerhard Walzl ${ }^{1}$

\begin{abstract}
Background: The global epidemiology of parasitic helminths and mycobacterial infections display extensive geographical overlap, especially in the rural and urban communities of developing countries. We investigated whether co-infection with the gastrointestinal tract-restricted helminth, Trichuris muris, and the intracellular bacterium, Mycobacterium bovis (M. bovis) $B C G$, would alter host immune responses to, or the pathological effect of, either infection.

Results: We demonstrate that both pathogens are capable of negatively affecting local and systemic immune responses towards each other by modifying cytokine phenotypes and by inducing general immune suppression. T. muris infection influenced non-specific and pathogen-specific immunity to M. bovis BCG by down-regulating pulmonary TH1 and Treg responses and inducing systemic $\mathrm{TH} 2$ responses. However, co-infection did not alter mycobacterial multiplication or dissemination and host pulmonary histopathology remained unaffected compared to BCG-only infected mice. Interestingly, prior M. bovis BCG infection significantly delayed helminth clearance and increased intestinal crypt cell proliferation in BALB/c mice. This was accompanied by a significant reduction in systemic helminth-specific TH1 and TH2 cytokine responses and significantly reduced local TH1 and TH2 responses in comparison to T. muris-only infected mice.

Conclusion: Our data demonstrate that co-infection with pathogens inducing opposing immune phenotypes, can have differential effects on compartmentalized host immune protection to either pathogen. In spite of local and systemic decreases in $\mathrm{TH} 1$ and increases in $\mathrm{TH} 2$ responses co-infected mice clear $\mathrm{M}$. bovis BCG at the same rate as BCG only infected animals, whereas prior mycobacterial infection initiates prolonged worm infestation in parallel to decreased pathogen-specific TH2 cytokine production.
\end{abstract}

Keywords: Helminth, Co-infection, Mycobacteria, Tuberculosis

\section{Background}

Tuberculosis (TB) is most prevalent in resource-poor countries and factors such as genetic susceptibility, malnutrition and circulating strain differences have been implicated as determinants of TB disease development in these regions $[1,2]$. Compelling evidence demonstrates

\footnotetext{
* Correspondence: hannonel@gmail.com; nelita@sun.ac.za

'Division of Molecular Biology and Human Genetics, MRC Centre for Molecular and Cellular Biology, NRF/DST Centre of Excellence in Biomedical TB Research, Faculty Medicine and Health Sciences, Stellenbosch University, Tygerberg, South Africa

${ }^{2}$ University of Queensland Diamantina Institute, Brisbane, QLD, Australia

Full list of author information is available at the end of the article
}

that many of these factors increase disease risk partly though the induction of host immune dysregulation and ultimately affect host control of Mycobacterium tuberculosis (M. tb) proliferation [3]. The high prevalence of parasitic helminth infections in TB affected communities, has highlighted co-infection as another risk factor compromising host immunity and thus a potential determinant for development of TB $[4,5]$. In support of this theory, several reports indicated that $\mathrm{TB}$ patients are commonly found to be co-infected with helminth species such as Trichuris trichiura and Ascaris lumbricoides [6] and present with increased total and helminthspecific serum immunoglobulin E (IgE) [7].

\section{Biomed Central}


Host control of mycobacterial or helminth infections largely rely on the induction of appropriately polarized immune responses. Protective immune responses to $M$. $t b$ infection are associated with enhanced T helper 1 (TH1) type cellular immunity and the production of characteristic TH1 cytokines such as tumor necrosis factor alpha (TNF- $\alpha$ ), interferon-gamma (IFN- $\gamma$ ) and interleukin-12 (IL-12) [8]. Conversely, protection against most helminths requires a $\mathrm{T}$ helper 2 ( $\mathrm{TH} 2$ ) type cellular immune response with production of distinct TH2 cytokines such as IL-4, IL-5, IL-13 and IL-9 [9,10]. Since TH1 and TH2 immune responses have the ability to concurrently downregulate each other, a state of co-infection could result in inappropriate protective host immune responses to either infections [11]. Furthermore, both pathogens have the potential to induce regulatory $\mathrm{T}$ cell (Treg) responses associated with production of immune suppressive cytokines such as IL-10 and transforming growth factor beta (TGF- $\beta$ ) [10-13].

In line with the TH1/TH2 dichotomy, hypotheses concerning helminth-mycobacterial co-infection postulate that a helminth-induced TH2 immune bias could inhibit development of protective cellular immune responses to $M$. $t b$, increase mycobacterial proliferation or lead to the failure of vaccine strategies against TB $[14,15]$. This theory is supported by numerous studies which have reported a reduction in $\mathrm{TH} 1$ responses to be associated with poor outcomes in TB patients [16] and latently infected individuals [17] with concurrent helminth infection. Helminthinduced regulatory (Treg) responses such as TGF- $\beta$ and IL-10 production have also been implicated in $S$. mansoniinduced progression to active TB of HIV-1 infected Ugandans [18]. It was also established that deworming of helminth-infected individuals restores cellular immune responses to mycobacterial purified protein derivatives (PPD) [19-21]. Similarly, deworming of helminth-infected Ethiopians immigrants in Israel resulted in increased cellular immune responses against HIV-and $M$. $t b$-specific antigens compared to untreated individuals [22], suggesting deteriorating immune responses and poor clinical outcomes in helminth-infected individuals might not be a result of inadequate nutrition or sanitation. Several reports have also indicated helminth-mediated modulation of vaccine responses. Children with prenatal sensitization to filariae and schistosomes were reported to display a down-regulation in TH1 responsiveness to BCG vaccination [23] and animal co-infection models have further demonstrated that a pre-existing infection with a lungmigrating helminth, can inhibit development of protective innate anti-TB responses by inducing the IL-4 receptor pathway and accumulation of alternatively activated macrophages [24]. In summary, most reports indicate that helminth infection significantly affects TB susceptibility. In contrast, very little data addressing the effect of mycobacterial infection on host immunity to helminth infections are available.

In the current study, we assessed the influence of coinfection on immune responses against the individual pathogens. We established a BALB/c co-infection model using Mycobacterium bovis (M. bovis) BCG and the gastrointestinal tract-restricted rodent helminth, Trichuris muris (T. muris) as TH1 and TH2 pathogenic assaults, respectively. The $M$. bovis BCG murine infection model is routinely used for studying anti-mycobacterial responses during latency as the associated immune response is similar to that induced during human $M . t b$ infection [25], whereas $T$. muris infection serves as a well described model for gastrointestinal tract restricted human soiltransmitted helminth (STH) infection [26]. We explored the possibility that concurrent infection with two pathogens, normally cleared by mice during single pathogen infection, might lead to mutually inhibitory immune dynamics and subsequent uncontrolled infection.

\section{Methods \\ Animals}

Specified pathogen free (SPF) female BALB/c mice (WT and IL-4 knock-out strains) between 6-8 weeks of age, were kept at the Faculty of Medicine and Health Sciences Animal Unit, Stellenbosch University (SU; South Africa) under conditions compatible with the SU guidelines for the care of animals. All procedures were approved by the SU Animal Ethics Board [Project license: 2003/186/p].

\section{Parasite enumeration and antigen preparation}

T. muris eggs were donated by Allison Bancroft (University of Manchester, UK). Egg propagation in BALB/c IL-4 knock-out mice (gift from Frank Brombacher, University of Cape Town, South Africa), helminth collection, and excretory/secretory (E/S) antigen preparations, were performed as described previously [27,28]. Helminth burdens were determined by quantification of intestinal adult worms by examining faecal matter under a dissection microscope. Mycobacterium bovis BCG Pasteur (donated by Robin Warren, SU, South Africa) was propagated to logarithmic growth phase in Middlebrook 7H9 (Difco) liquid culture, supplemented with $0.2 \%$ glycerol, $0.05 \%$ Tween 80 and 10\% albumin-dextrose-catalase (ADC, Merck) at $37^{\circ} \mathrm{C}$. Bacterial proliferation was assessed by manual counting of colony forming units (CFU) from serial dilutions of homogenized lungs and spleens, plated on Middelbrook 7H11 (Difco) agar plates supplemented with $0.2 \%$ glycerol and $10 \%$ oleic acid-albumin-dextrosecatalase (OADC, BD Biosciences).

\section{Co-infection protocol}

Two infection protocols were used during this study. Each experiment consisted of 3 groups of 5-10 animals 
per group. Groups included M. bovis BCG-T. muris coinfected, BCG-only infected and T. muris-only infected mice. The first protocol (Figure 1A) was intended to establish a chronic, low grade $M$. bovis BCG infection that was subsequently followed by a TH2-inducing T. muris infection. Mice were infected intranasally (i.n.) with $1-5 \times 10^{5} \mathrm{CFU}$ BCG bacilli per mouse or an equal volume of PBS. Briefly, mice were lightly anesthetized by intraperitoneal (i.p.) injection of a $200 \mu \mathrm{l}$ mixture consisting of Ketamine (12 mg/ml Anaket-V, Centaur Labs) and Xylazine $(1.6 \mathrm{mg} / \mathrm{ml}$, Rompun, Bayer). Mice were gently lifted by the loose skin at the throat, and kept upright with its head tilted back and the nose pointed up. Using a pipette with a sterile tip, $40 \mu \mathrm{l}$ of the declumped mycobacterial suspension was applied to the nostrils. Animals were maintained upright for another 30 seconds to ensure complete delivery to the respiratory system. Six weeks (day 42) later, mice were infected under light anaesthesia intragastrically (i.g.) with 200-250 (low dose) or 500-600 (high dose) embryonated T. muris eggs or an equal volume of PBS. At week 9 (day 63), mice were culled and the

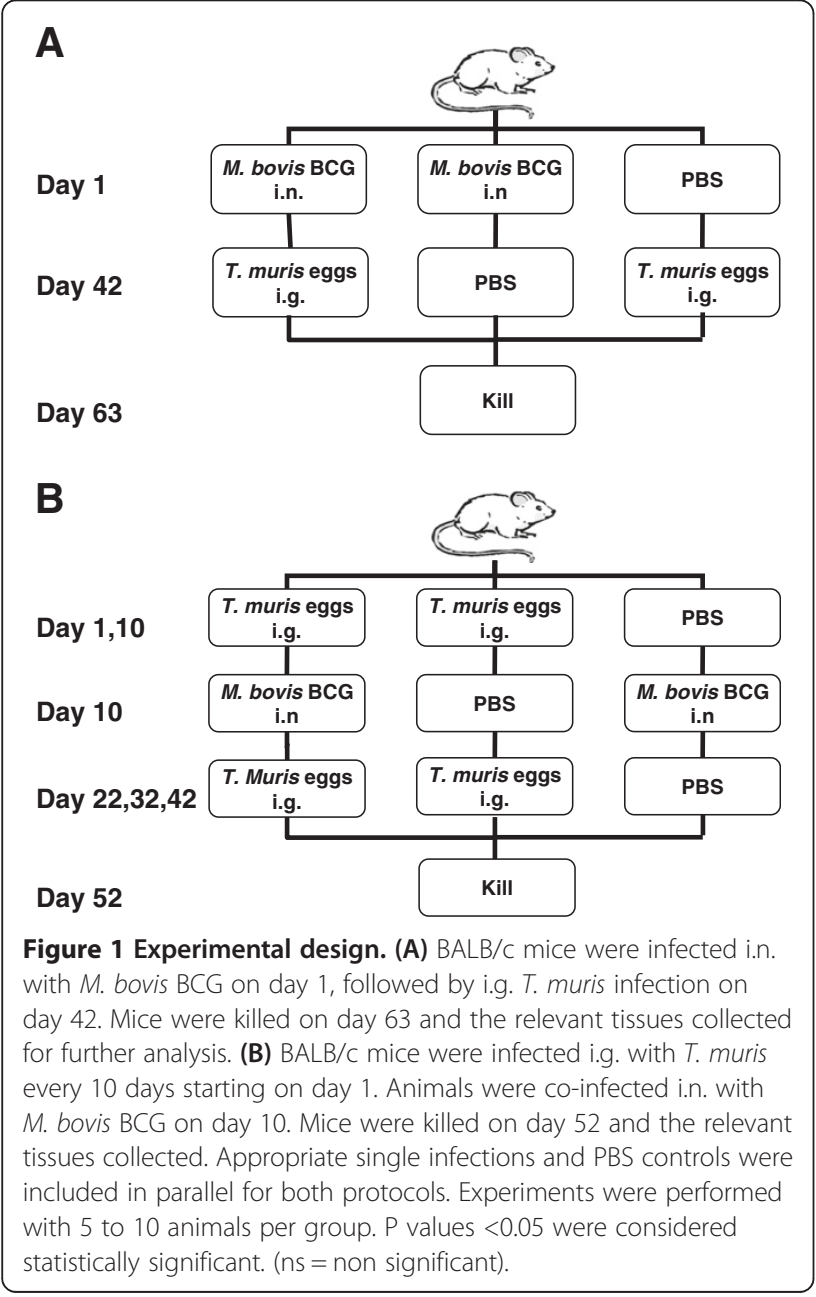

relevant organs removed for investigation. The second protocol (Figure 1B) was designed to first establish a TH2-inducing T. muris infection prior to challenge with M. bovis BCG infection. Animals were infected i.g. with 200-250 embryonated T. muris eggs or an equal amount of PBS on day 1 and every 10 days thereafter until experimental completion. On day 10, animals were infected i.n. with $1-5 \times 10^{5}$ CFU BCG bacilli or an equal volume of PBS. After 6 weeks (day 52), all mice were humanely euthanized and the relevant organs removed for investigation. Experiments were completed in triplicate at three separate times.

\section{Immune phenotyping and intracellular cytokine analysis}

Immune phenotyping was performed using single cell suspensions from spleens and mesenteric lymph nodes (MLNs). Intracellular cytokine expression was determined following stimulation with $50 \mathrm{ng} / \mathrm{ml}$ Phorbol 12-myristate 13-acetate (PMA) (Sigma), $1 \mu \mathrm{g} / \mathrm{ml}$ Ionomycin (Sigma) and $10 \mu \mathrm{g} / \mathrm{ml}$ Brefeldin A (BFA) (Sigma) for 4 hours at $37^{\circ} \mathrm{C}$ and $5 \% \mathrm{CO}_{2}$. Cells were resuspended in PBS containing $1 \%$ BSA and $0.1 \%$ Sodium Azide (wash buffer) and stained for 30 minutes with fluorochrome conjugated anti-mouse antibodies against CD3, CD4, CD8, CD25, B220, Foxp3, IFN- $\gamma$ and IL-4 (BD Biosciences, Caltag or Biolegend). Cells were fixed with $1 \%$ formaldehyde, washed and resuspended in wash buffer. Lymphocyte populations were determined based on their Forward/ Side scatter profile and gates set with the help of appropriate FMOs and Isotype controls. Acquisitions were performed on a FACSCalibur (BD Biosciences) using appropriate instrument settings, color compensation and isotype controls for all antibodies. At least $5 \times 10^{4}$ lymphocyte events were acquired and data analysis performed using CellQuest software (BD Bioscience).

\section{In vitro pathogen-specific cytokine analysis}

Spleen $\left(1 \times 10^{7}\right.$ cells $\left./ \mathrm{ml}\right)$ single cell suspensions were stimulated for 24 hours with live BCG cultures (MOI 5:1), $50 \mu \mathrm{g} / \mathrm{ml} \mathrm{E} / \mathrm{S}$ antigen or culture media as control at $37^{\circ} \mathrm{C}$, $5 \% \mathrm{CO}_{2}$. Culture supernatants were used for cytokine concentration analyses using the luminex bead-array technology (LINCO Research) to test for the soluble cytokines IFN- $\gamma$, TNF- $\alpha$, IL-4, IL-10, IL-13 and IL-17 using a BioPlex platform (Bio-Rad Laboratories). Background readings were controlled by subtraction of unstimulated control sample measurements. Values were checked against internal quality controls to monitor analysis accuracy within specified concentration ranges.

\section{Nucleic acid extraction and relative quantitative real time PCR}

Total RNA was extracted from the upper right lobe of mouse lungs and spleen tips using Trizol (Gibco BRL) 
and subsequently treated with a DNA-free kit (Ambion) to remove contaminating DNA. First strand cDNA was transcribed using the QuantiTect Reverse Transcription kit (Qiagen) according to the manufacturer's protocols. Relative quantification of IFN- $\gamma$, IL-4, IL-10, TGF- $\beta$ and Foxp3 were performed using SYBR Green PCR Master Mix kit (Roche), cDNA $(500 \mu \mathrm{g})$ and primers $(0.5 \mu \mathrm{M})$ on the LightCycler system v3.5 (Roche). All primers were designed to span intron-exon boundaries (Table 1). The delta-delta $\mathrm{Ct}$ method was used to calculate relative gene expression levels between two samples. Gene expression was assayed quantitatively and normalized to that of a housekeeping gene (GAPDH, HPRT, 18S-RNA) to obtain a RNA ratio in order to establish the relevant change in RNA expression [29].

\section{Histology}

Left upper lung lobes were fixed in 10\% buffered formalin, embedded in paraffin blocks and sections (3-5 $\mu \mathrm{m})$ stained with Haematoxylin and Eosin (H\&E) for light microscopy. Pulmonary histopathological scoring was performed in a blinded fashion and calculated separately for each lung section as previously described [32]. In brief, a scale of 0 to 4 was used to individually score the level of peribronchiolitis, perivasculitis, interstitial pneumonitis and alveolitis of each section in order to obtain an average score for each lung. A score of 0 was based upon observation of normal, uninfected mouse lung samples and a score of 4 on previous studies of greatest inflammatory change and pathology brought about by i.n M. bovis BCG infection in BALB/C mice. Scoring of gastrointestinal histopathology was achieved by measuring mucus production, presence of mast cells and mitotic body enumeration in fixed caecum tips imbedded in paraffin blocks. Sections (3-5 $\mu \mathrm{m})$ were used for Periodic Acid Schiff (PAS) staining to score goblet cell-mucus production within caecal crypts as the percentage PAS positive stain in the crypt epithelium and lamina propria. Acidified toluidine blue staining was used for the quantification of mast cells in caecum tip samples and enumeration of mitotic bodies within caecum crypts. Scoring was conducted from two sets (cross sectional and longitudinal) of 20 caecal crypt units per animal. All slides were evaluated using the ZS300 Imaging system v.3.0 (Carl Zeiss Vision).

\section{Statistical analysis}

Data was analyzed using STATISTCA v.7 (StatSoft) software. Nonparametric analysis and Mann-Whitney U tests were performed for comparison between groups and the data presented as median values. Multiple group analysis included the multiple comparison correction (Bonferroni). Statistically significant differences were judged as $\mathrm{p} \leq 0.05$.

\section{Results}

M. bovis BCG clearance and lung pathology is not influenced by an established or successive $T$. muris infection

The influence of $T$. muris infection on host ability to control a chronic, low grade $M$. bovis BCG infection in $\mathrm{BALB} / \mathrm{c}$ mice was investigated for both experimental protocols (Figure 1A and B). Results demonstrated that an ongoing helminth-induced TH2 immune background, pre-established by $T$. muris trickle infection, failed to alter mycobacterial proliferation and dissemination when compared to $M$. bovis BCG-only infected mice in the lungs (Figure 2A) and spleen (data not shown). Similarly, initiation of a TH2 immune environment subsequent to BCG infection, resulted in equivalent pulmonary bacterial burdens between co-infected and BCG-only infected groups (Figure $2 \mathrm{~B}$ ). These end point $\mathrm{CFU}$ findings were confirmed by growth curve data demonstrating no significant difference in pulmonary mycobacterial burden between co-infected and M. bovis BCG-only infected mice at several time points post $M$. bovis BCG infection (Figure 2C).

Table 1 List of primer sequences used for relative quantitative real-time PCR

\begin{tabular}{lll}
\hline Target & Forward & Reverse \\
\hline HPRT & GACTGTAGATTTATCAGACT & GTCTGGCCTGTATCCAACACTTC \\
GPDH & GGTGGCAGAGGCCTTTG & TGCCGATTTAGCATCTCCTT \\
${ }^{*} 18 \mathrm{~S}[30]$ & GTCTGTGATGCCCTTAGATG & AGCTTATGACCCGCACTTAC \\
${ }^{*}$ TGF- $\beta[30]$ & CCGCAACAACGCCATCTATG & CTCTGCACGGGACAGCAAT \\
${ }^{*}$ IFN-y [31] & AAGTTCTGGGCTTCTCCTCCTG & GCCAGTTCCTCCAGATATCCAAGA \\
${ }^{*}$ IL-10 [30] & CTGGACAACATACTGCTAACCG & GGGCATCACTTCTACCAGGTAA \\
${ }^{*}$ IL-4 [31] & TCAACCCCCAGCTAGTTGTC & TTCAAGCATGGAGTTTCCC \\
GATA3 & CTGGAGGAGGAACGCTAATG & GGTTGAAGGAGCTGCTCTTG \\
${ }^{T}$ Tbet & AGCAAGGACGGCGAATGTT & GGGTGGACATATAAGCGGTTC \\
${ }^{*}$ Foxp3 [30] & CACAATATGCGACCCCCTTTC & AACATGCGAGTAAACCAATGGTA \\
\hline
\end{tabular}

${ }^{*}$ Primer sequences adapted from reference. 


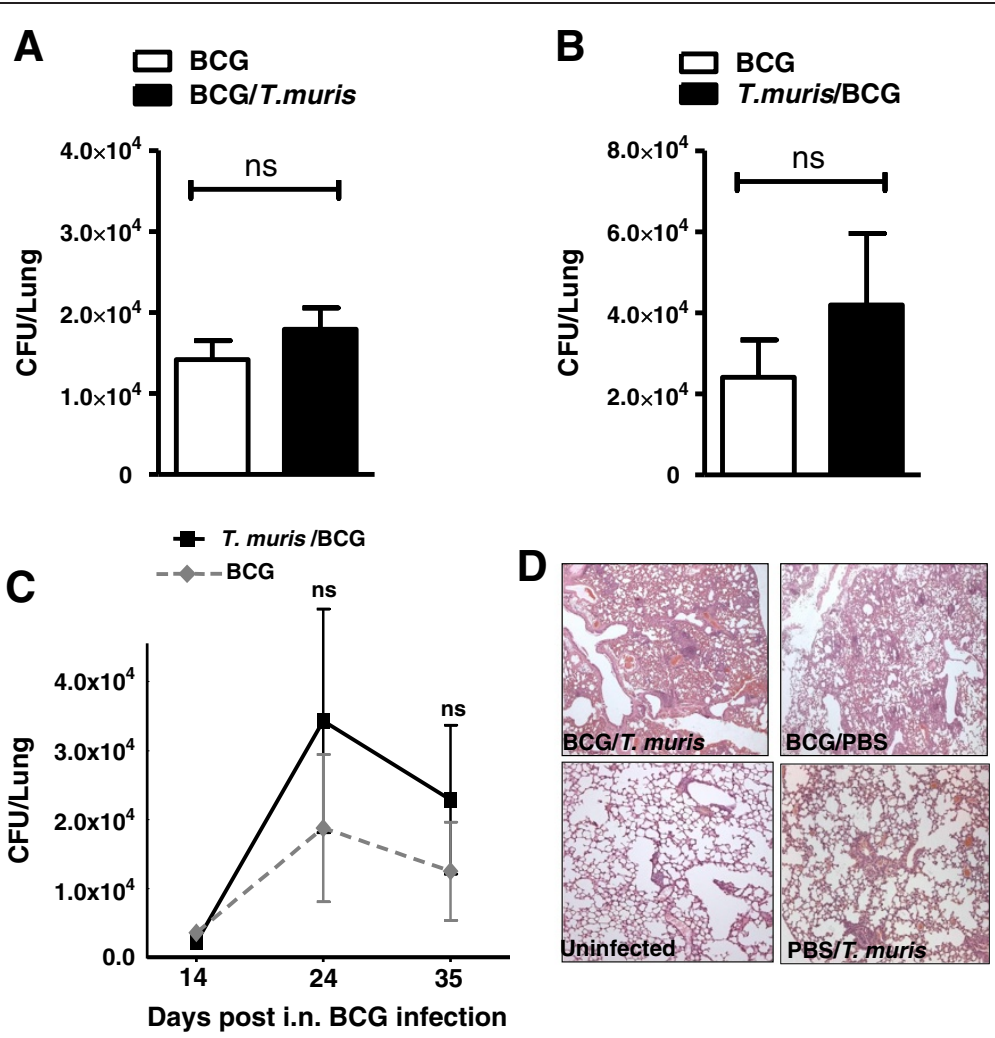

E
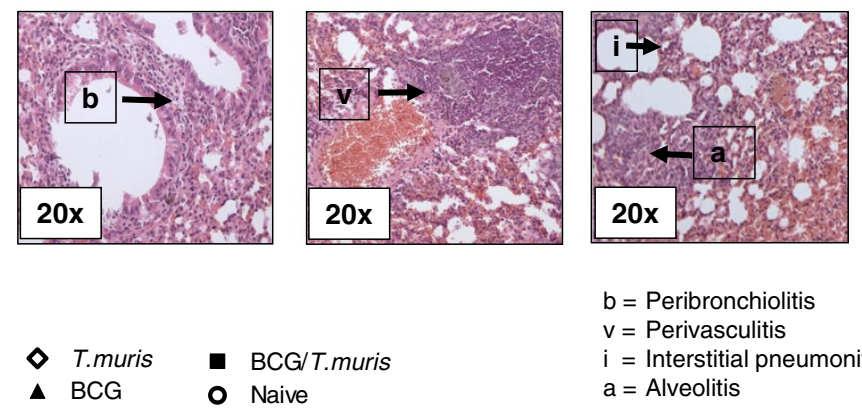

$\mathrm{b}=$ Peribronchiolitis

$\mathrm{v}=$ Perivasculitis

$\mathrm{i}=$ Interstitial pneumonitis

- BCG

$\mathrm{a}=$ Alveolitis

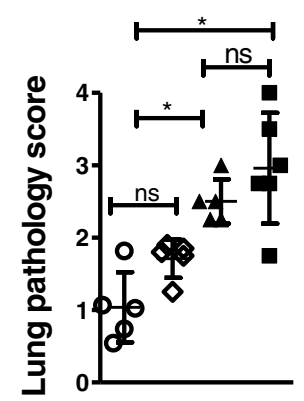

Figure 2 (See legend on next page.) 
(See figure on previous page.)

Figure $2 M$. bovis BCG clearance and mycobacterial-induced lung pathology is not influenced by an established or successive $T$. muris infection. (A) Viable pulmonary M. bovis BCG CFU numbers at experimental endpoint in co-infected (black) and BCG-only (clear) infected BALB/C mice infected according to experimental design as shown in Figure 1A. Data display mean \pm SEM, representing 3 individual experiments of 5-6 animals per group. (B) Viable pulmonary M. bovis BCG CFU numbers at experimental endpoint in co-infected (black) and BCG-only (clear) infected $\mathrm{BALB} / \mathrm{C}$ mice infected according to experimental design as shown in Figure 1B. Data display mean \pm SEM, representing 3 individual experiments of 5-6 animals per group. (C) Viable pulmonary M. bovis BCG CFU growth curve data of co-infected (black) and BCG-only (clear) infected mice at days 14, 24 and 35 post BCG infection (D) Representative histological H\&E stained lung sections captured at 10x magnification illustrating the differences in histopathology between BCG/T.muris co-infected, BCG-only infected, uninfected and T. muris-only infected BALB/C mice infected according to experimental design as shown in Figure 1A. (E) Pulmonary histopathological scoring was performed in a blinded fashion according to the degree of peribronchiolitis (b), perivasculitis (v), interstitial pneumonitis (i) and alveolitis (a) per lung. Average pulmonary scores of BALB/C mice infected according to experimental design as shown in Figure 1A. Groups included naive (circle), T. muris-only (diamond), BCG-only (triangle) and co-infected (square) mice. Data display mean \pm SD, representing 2 individual experiments of 5-6 animals per group. P values $<0.05$ were considered statistically significant. ( ${ }^{*} p \leq 0.05$, ns $=$ non significant).

Histological scoring of both infection protocols indicated that $T$. muris-only infected mice displayed normal lung pathology with only minimal cell infiltration compared to naive mice, whereas the degree of pulmonary pathology and the cellular composition and organization in the lungs following $M$. bovis BCG co-infection were significantly increased (Figure $2 \mathrm{D}$ and $\mathrm{E}$ ). No significant differences in pulmonary inflammatory scores could be detected between BCG-only and co-infected mice for either infection protocols (Figure 2D, 2E and Additional file 1: Figure S1).

\section{Previously established BCG infection delays $T$. muris expulsion in co-infected animals}

The influence of $M$. bovis BCG co-infection on eradication of T. muris in BALB/c mice was evaluated as worm expulsion for both experimental protocols (Figure 1A and $\mathrm{B})$. In each case, susceptible IL-4KO mice with disrupted protective $\mathrm{TH} 2$ responses, were included as controls of delayed worm clearance [33]. Following the infection strategy in Figure 1A, the helminth burden at experimental completion demonstrated that almost half $(44 \% ; 4 / 9)$ of mice with an established chronic BCG infection, that were subsequently co-infected with a low dose of helminth eggs, still presented with $T$. muris, whereas significantly more animals $(88 \% ; 7 / 8)$ from the T. muris-only infected group had cleared all helminths (Figure 3A). Both groups displayed significantly lower worm burdens compared to IL-4KO mice infected with T. muris only (Figure 3A). Similar results were observed in experimental repeats using a high dose of helminth eggs, showing helminth clearance in (100\%; 0/9) T. murisonly infected BALB/c mice, whereas $T$. muris expulsion failed in $(40 \% ; 4 / 10) M$. bovis BCG co-infected BALB/c mice (Figure $3 \mathrm{~B}$ ). However, when the infection sequence was reversed, where an initial $T$. muris infection was followed by a subsequent BCG infection (Figure 1B), repeat experiments consistently indicated helminth clearance in $>90 \%$ of both co-infected and T. muris-only infected mice (data not shown).
Co-infection exacerbates cell proliferation in caecum tips A striking observation was the massive amount of mucus present in the caeca and colons of mice co-infected according to either experimental protocol (Figure 1A and B) in comparison to T. muris-only infected mice. Although PAS stained samples failed to demonstrate significant differences in goblet cell formation or caecal crypt-mucus production between co-infected and $T$. muris-only infected mice (Figure 4A), acidified toluidine blue staining showed significantly increased numbers of mitotic figures in caecum crypts of co-infected animals as identified by their dense chromatic structure (Figure 4B). Very few mast cells were observed within the epithelium or lamina propria of the crypt units in co-infected mice and no significant statistical differences in mast cell recruitment were observed between infection groups (Figure 4C).

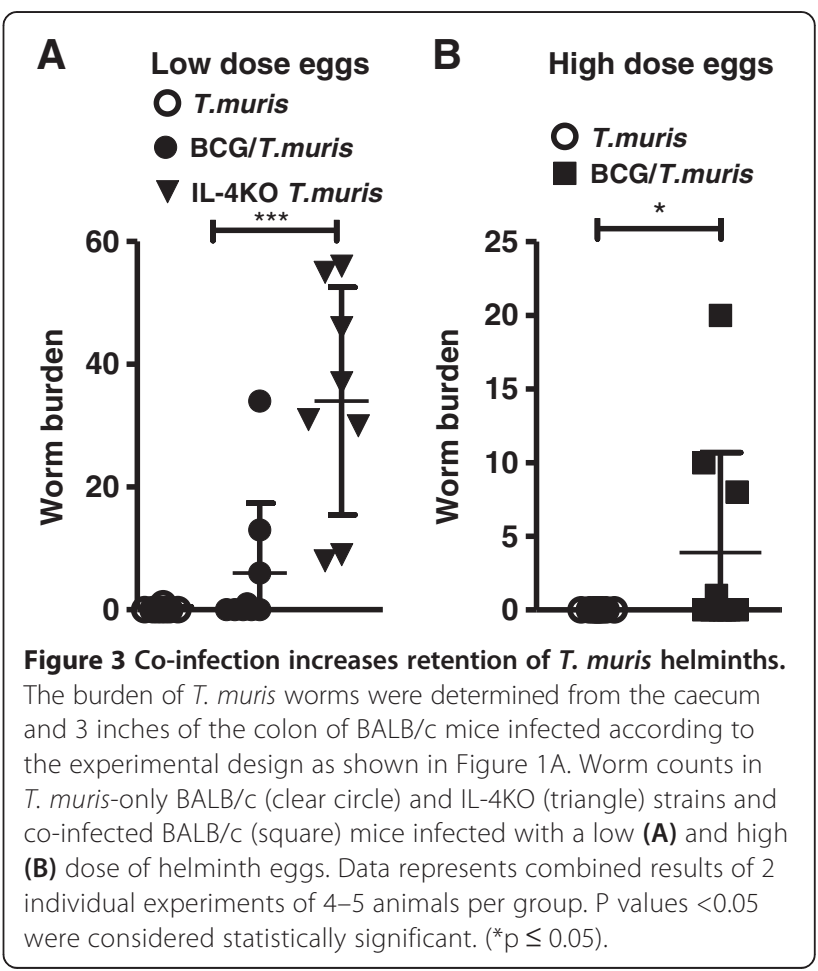


A
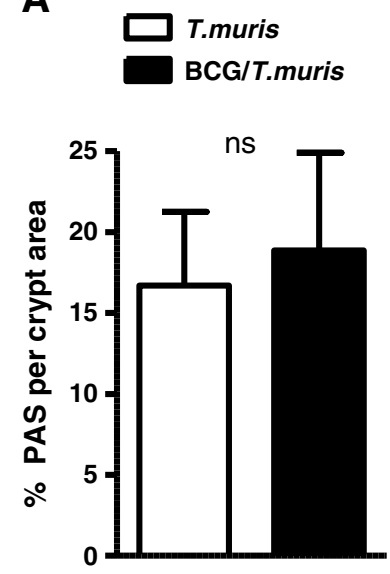

B

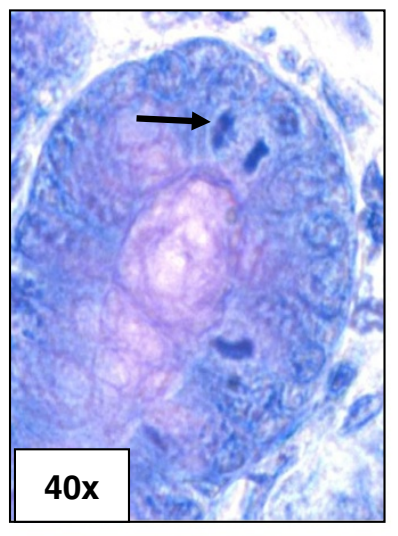

T.muris

BCG/T.muris

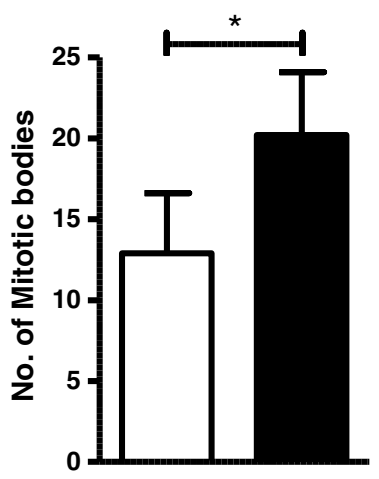

C
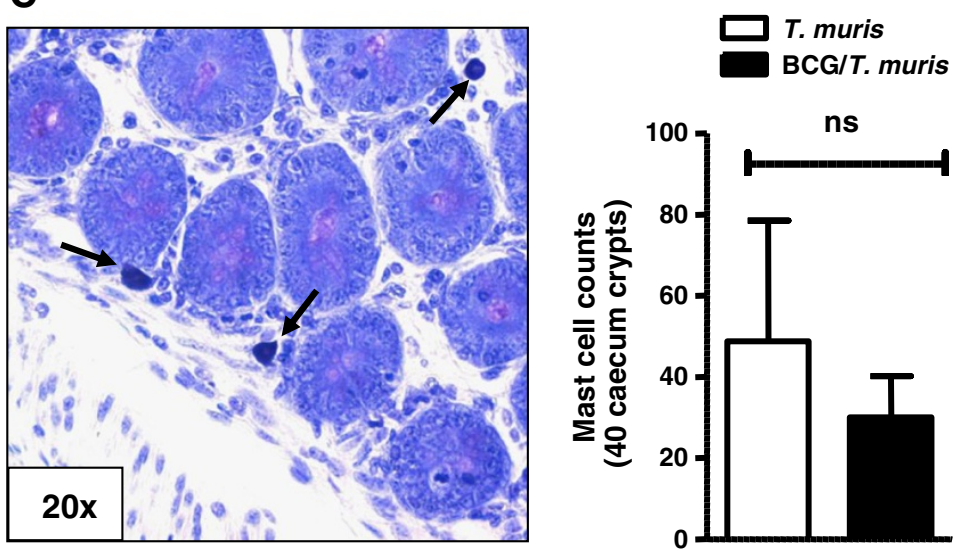

Figure 4 Co-infection increases mitotic figures in the caecum crypts. (A) Histological analysis of goblet cell numbers as determined by the percentage $\mathrm{PAS}^{+}$cells (indicated by arrow) per $2 \times 20$ cross sectional crypt units in T. muris-only (clear) and co-infected (black) BALB/c mice infected according to the experimental design as shown in Figure 1A. Data display median \pm min-max, representing 2-3 individual experiments of 5 animals per group. (B) Toluidine blue stained mitotic bodies (indicated by the arrows) were counted in $2 \times 20$ crypts/slide. Numbers of mitotic bodies as determined from cross-sectional and longitudinal crypt units in co-infected (black) and T. muris-only (clear) infected BALB/c mice infected according to Figure 1A. Data display median \pm min-max, representing 2-3 individual experiments of 5 animals per group (C) Toluidine blue staining for the assessment of mast cells (indicated by arrows) in cross sectional and longitudinal crypt units demonstrated few mast cells within the lamina propria and crypt epithelium of the caecum tissue with most mast cells residing within the submucosa surrounding the caecum. Bar graph indicating the numbers of mast cells measured in co-infected (black) and T. muris-only (clear) infected groups per 40 caecum crypts. Data display median \pm SD of 5 animals per group. P values $<0.05$ were considered statistically significant. (ns $=$ non significant). 


\section{Co-infection increases $\mathrm{CD}^{+}$splenocyte frequencies and modifies the TH1/TH2 immune balance}

Flow cytometric analysis demonstrated that co-infection according to either infection protocol (Figure 1A and B) did not impact lymphocyte composition in the spleen or MLN, since no significant differences between infection groups were observed for populations of $\mathrm{CD}^{+} \mathrm{T}$ cells

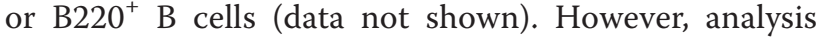
of ex-vivo lymphocyte subpopulations in BALB/c mice infected according to Figure 1A, revealed an increase in $\mathrm{CD}^{+} \mathrm{T}$ helper cell population in the spleens of mice co-infected according to the protocol in Figure 1A, when compared to BCG-only infected mice (Figure 5A). Although no differences in the percentages of natural regulatory $\mathrm{T}$ cell $\left(\mathrm{CD} 4^{+} \mathrm{CD} 25^{+} \mathrm{Foxp}^{+}\right)$populations were observed between infection groups in either the spleen or MLN (data not shown), co-infection significantly increased the percentage of IL-4-producing $\mathrm{CD}^{+}$and $\mathrm{CD}^{+}$splenocytes in comparison to $M$. bovis BCG-only infected controls (Figure 5B). IL-4-producing $\mathrm{CD}^{+}$and $\mathrm{CD}^{+} \mathrm{MLN}$ cells from co-infected mice were however significantly reduced in comparison to T. muris-only infected mice (Figure 5C). A marked decrease in $\mathrm{CD}^{+} \mathrm{IFN}^{+}$ MLN cells was also observed in co-infected mice in comparison to mice infected only with $T$. muris, whereas frequencies of $\mathrm{CD}^{+}{ }^{+} \mathrm{IFN} \gamma^{+}$MLN cells were measured at similar levels between co-infected and T.muris-only infected mice (Figure 5D).

When the infection order was reversed during trickle infection to address the effect of introduction of co-infection with $M$. bovis BCG into an established helminth-induced TH2 environment (Figure 1B), a significant increase in activated effector $\mathrm{T}$ cell $\left(\mathrm{CD} 4^{+} \mathrm{CD} 25^{+}\right.$Foxp $\left.3^{-}\right)$percentages in MLNs of co-infected animals was observed in comparison to $T$. muris-only infected controls (Figure 5E). A trend towards decreased frequencies of inducible regulatory $\mathrm{T}$ cells (iTreg) $\left(\mathrm{CD}^{+}{ }^{+} \mathrm{CD} 25^{-}\right.$Foxp $^{+}$) was also observed in the MLNs of co-infected compared to T. muris-only infected mice (Figure 5F). No significant differences in ex vivo cytokine production between infection groups were observed for $\mathrm{CD}^{+}$and $\mathrm{CD}^{+}$lymphocytes in the spleen or MLNs (data not shown).

\section{Co-infection reduces pathogen-specific $\mathrm{TH} 1$ and $\mathrm{TH} 2$ immune responses}

Pathogen-specific TH1/TH2/TH17/Treg cytokine immune responses in the spleen were analyzed only in BALB/c mice infected according to the protocol in Figure 1A, since no significant differences in ex vivo T cell cytokine production between infection groups were observed in the spleens or lungs of mice infected according to the protocol in Figure 1B.

E/S stimulated splenocytes from both co-infected and BCG-only infected mice displayed a prominent reduction in TH2/Treg (IL-4, IL-13 and IL-10) cytokine production when compared to $T$. muris-only infected animals, although IL-4 levels were significantly increased in coinfected compared to BCG-only infected mice (Figure 6A). Similarly, E/S-specific TH1 cytokines (TNF- $\alpha$ and IFN- $\gamma$ ) were reduced in both the co-infected and BCG-only infected groups with respect to $T$. muris-only infected animals (Figure 6A). No notable differences between the infection groups were observed for helminth-specific IL-17 production (data not shown).

BCG-stimulated splenocytes displayed notably low concentrations of TH2 (IL-4 and IL-13) cytokines in all infection groups. Although no significant differences in concentrations of the cytokines, IFN- $\gamma$ and IL-17 (Figure 6B) were measured between infection groups, co-infection significantly decreased production of the cytokines TNF- $\alpha$, IL-10 and IL-4 in comparison to T. murisonly and/or BCG-only infected mice (Figure 6B).

\section{Co-infection reduces the pulmonary cytokine gene expression profile relative to BCG-only infected animals}

To assess whether the immunological changes observed in mice infected according to the infection protocol indicated in Figure 1A, also extends to alterations in pulmonary and splenic gene expression levels, the relative gene expression of co-infected mice and BCG-only infected mice was determined. At week 9, the relative gene expression ratios from co-infected mice demonstrated significantly decreased RNA levels in the lungs for TGF$\beta(p=0.034)$, Foxp3 $(p=0.042)$ and IFN- $\gamma(p=0.012)$ relative to BCG-only infected mice (Figure 7$)$. The levels of IL-10 ( $\mathrm{p}=0.072)$ also showed a trend towards decreased expression across these two groups (Figure 7). Analysis of RNA profiles in the spleen failed to show significant variations in expression levels for any of the genes measured, between co-infected and BCG-only infected groups (data not shown).

\section{Discussion}

In this study, we demonstrate the capability of the gastrointestinal tract restricted helminth, $T$. muris, to induce local and systemic $\mathrm{TH} 2$ immune responses that affect immunity to $M$. bovis BCG. Of particular interest was the significant reduction in BCG-specific TNF- $\alpha$ and IL-10 cytokine concentrations and significant increase in IL4-producing $\mathrm{CD}^{+}$and $\mathrm{CD}^{+} \mathrm{T}$ cells in the spleens of co-infected mice, in comparison to BCG-only infected mice. In addition, we show that co-infection significantly reduced pulmonary IFN- $\gamma$, TGF- $\beta$ and Foxp3 gene expression, relative to BCG-only infected mice. Collectively, our data show a down-regulation in pulmonary $\mathrm{TH} 1$ and Treg-associated responses and the induction of systemic $\mathrm{TH} 2$ responsiveness following co-infection. Nevertheless, lung and systemic bacterial 

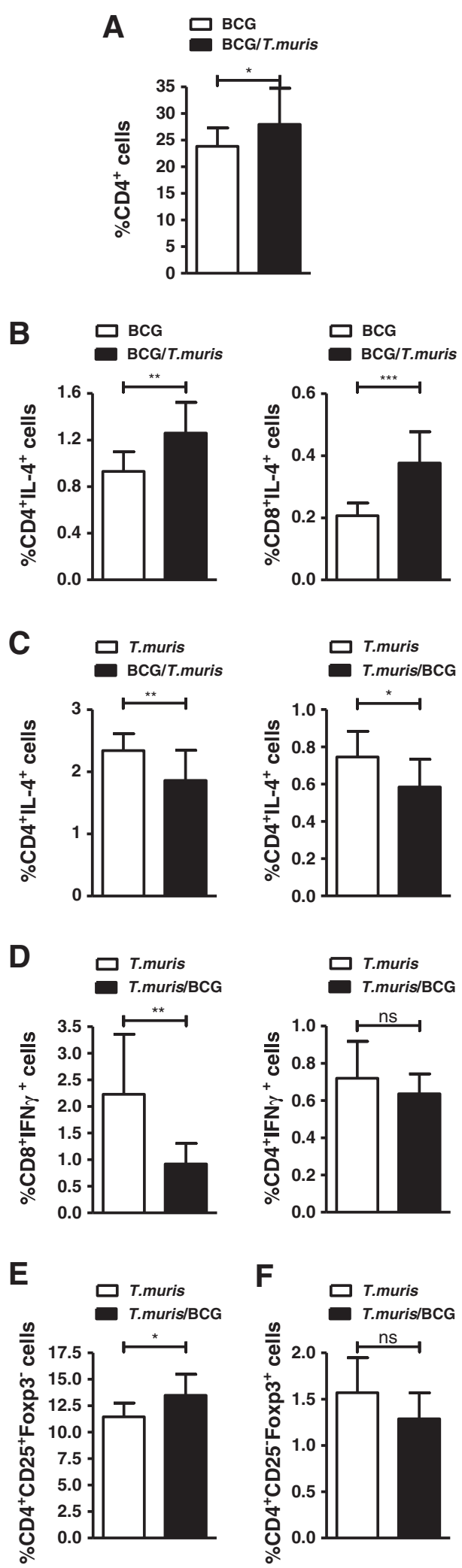

Figure 5 Co-infection affects the frequency of $\mathrm{CD} 4^{+}$and Treg lymphocyte populations and alters ex vivo $\mathrm{TH} 1 / \mathrm{TH} 2$ cell populations. (A) Percentages of $\mathrm{CD}^{+}$splenocytes in BCG-only (clear) and co-infected (black) BALB/c mice infected according to experimental design in Figure 1A. Data display median \pm min-max, representing 2-3 individual experiments of 20 animals per group. (B) Percentages of $\mathrm{IL}-4$ producing $\mathrm{CD}^{+}$and $\mathrm{CD} 8^{+}$splenocytes in BCG-only (clear) and co-infected (black) BALB/c mice infected according to the protocol in Figure 1B. Data display median \pm min-max, representing 2-3 individual experiments of 8-10 animals per group. (C-D) Percentages of $\mathrm{CD} 4^{+} \mathrm{IL}-4^{+}, \mathrm{CD} 8^{+} \mathrm{IL}-4^{+}$and $\mathrm{CD} 4^{+} \mathrm{IFN}-\gamma^{+} \mathrm{MLN}$ cell populations in T. muris-only (clear) and co-infected (black) BALB/c mice infected according to experimental design in Figure 1B. Data represents experiments with 8-10 animals per group. Percentages of $(\mathbf{E})$ activated T cells (CD4 $\left.{ }^{+} \mathrm{CD}^{2} 5^{+} \mathrm{Foxp}^{-}\right)$and $(\mathbf{F})$ inducible regulatory $\mathrm{T}$ cells (iTreg) $\left(\mathrm{CD}^{+} \mathrm{CD} 25 \mathrm{Foxp}^{+}\right)$in MLNs of T. muris-only (clear) and co-infected (black) BALB/c mice infected according to experimental design in Figure 1B. Data display median \pm min-max, representing $2-3$ individual experiments of 8-10 animals per group. $P$ values $<0.05$ were considered statistically significant. ${ }^{*} p \leq 0.05,{ }^{* *} p \leq 0.01$, ns $=$ non-significant).

burdens remained unaffected in co-infected mice and did not translate into alterations in pulmonary histopathology with respect to BCG-only infected mice, suggesting that protective host immune responses could be sufficiently compartmentalized to appropriately respond to the mycobacterial infection. Previous reports have demonstrated the host's ability to fully compartmentalize immunity during co-infection with $\mathrm{TH} 1$ and $\mathrm{TH} 2$-inducing pathogens at different sites of the mammalian body [34]. While helminth co-infection has been shown to negatively influence host control of other intracellular pathogens, several reports suggest that this outcome is specific to the helminth species investigated [35-38]. Even so, T. muris infection marginally increased pulmonary cellular infiltration with respect to naive mice, likely due to systemic inflammation caused by the helminth infection or the presence of helminth antigens. Although not discussed here, work done by us shows that neither adoptive transfer of splenocytes or MLN leukocytes from helminth-only infected animals, or abrogation of IL- 4 in IL- 4 deficient mice, resulted in altered mycobacterial burden (unpublished data). These transfer experiments could however not exclude a role for suppressive MLN or spleen cell subsets since purified populations were not used in these experiments. Also, the timing of transfer and the absence of continual pathogen-derived antigen stimulation in the recipient host could play a role in the effector responses and activation status of these cells.

Interestingly, our results show that prior pulmonary $M$. bovis BCG infection also significantly affected local and systemic protective host immune responses to a subsequent $T$. muris infection. Although the lack of ex vivo phenotyping data from BCG-only infected mice is a weakness in this infection protocol, co-infected mice displayed a significant reduction in E/S-specific TH1 

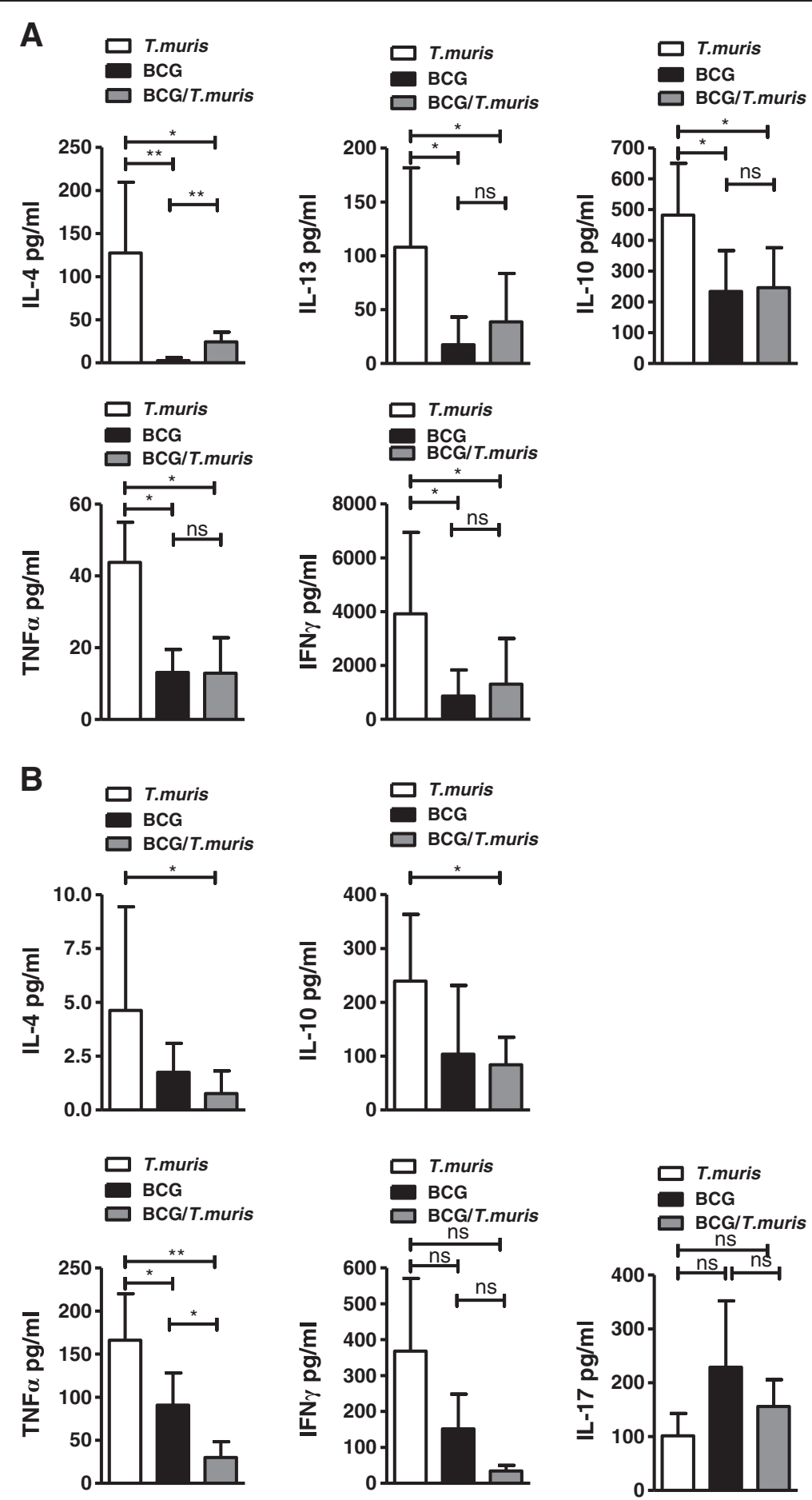

Figure 6 Co-infection leads to altered pathogen-specific TH1 and TH2 immune responses. TH1 and TH2 cytokine concentrations were measured from 24 hour (A) E/S stimulated and (B) BCG-stimulated splenocyte cultures of co-infected (grey), T. muris-only (clear) and BCG-only (black) BALB/c mice infected according to the protocol illustrated in Figure 1A. Results from stimulated values were corrected for background unstimulated controls. Data display median \pm min-max, representing $2-3$ individual experiments of 5 animals per group. P values $<0.05$ were considered statistically significant. ( ${ }^{*} p \leq 0.05,{ }^{* *} p \leq 0.01, n s=$ non-significant). 


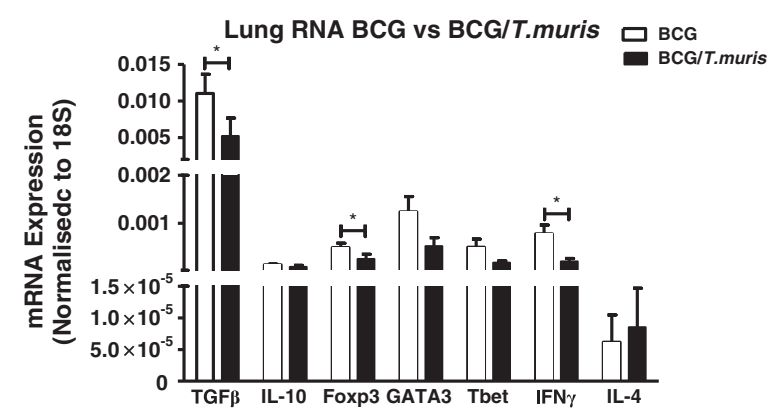

Figure 7 Co-infection decreases the expression ratio of pulmonary RNA cytokine transcripts relative to those of BCG-only infected BALB/c mice. BALB/c mice were co-infected (black) according to the protocol illustrated in Figure $1 \mathrm{~A}$ with BCG-only (clear) infected mice included as controls. At week 9, total RNA was extracted from the right upper lung lobe, cDNA produced and the relative gene expression ratio in co-infected mice relative to that of BCG-only infected mice, determined by real-time PCR. Following HKG normalization and delta-delta $\mathrm{Ct}$ analysis, the expression ratio of the genes TGF- $\beta$, IL-10, Foxp3, GATA3, T-bet, IFN- $\gamma$ were calculated. Data display median \pm SE, representing 8-10 animals per group. $\mathrm{P}$ values $<0.05$ were considered statistically significant in comparison to BCG-only infected. $\left({ }^{*}=p<0.05\right)$.

and $\mathrm{TH} 2$ cytokine responses in the spleen, and significantly reduced $\mathrm{IL}-4$ producing $\mathrm{CD}^{+}$and $\mathrm{CD}^{+} \mathrm{T}$ cells and IFN- $\gamma$-producing $\mathrm{CD}^{+} \mathrm{T}$ cells in the mesenteric lymph nodes when compared to $T$. muris-only infected mice. In support of a functional role for this reduction in T. muris-specific immunity, we demonstrated an associated delay in helminth clearance and increased helminthrelated intestinal pathology in co-infected mice, when compared to T. muris-only infected mice. These intestinal pathological changes were characterized by increased cell turnover, suggesting increased apoptosis or cell damage, necessitating cell replacement [39]. Intestinal crypt cell apoptosis was previously reported to occur following $T$. muris infection and subsequently shown to be reduced following neutralization of IFN- $\gamma$ and TNF- $\alpha$ [40]. In parallel with this we observed an increase in intestinal mucus production, which likely operates as a compensatory mechanism to aide expulsion of persisting parasites. Our results verify reports illustrating that $M$. bovis co-infection increase helminth parasite burden and correlates with decreased IL-4 and IL-13 cytokine production [41]. Our findings also agree with early reports demonstrating a reduction in protective immune responses and a delay in T. muris expulsion during other co-infections with Nematospiroides dubius, Plasmodium berghei or Trypanosoma brucei [42-44]. It is well established that resolution of T. muris infection is characterized by the production of TH2 cytokines, resulting in intestinal goblet cell hyperplasia and increased intestinal epithelial cell turnover $[45,46]$. On the other hand, mast cells, $\gamma \delta$ T cells and eosinophils are suggested as dispensable for T. muris expulsion [45,47] and the contribution of $\mathrm{B}$ cells and antibody responses remains controversial [48-50]. Previous reports convincingly show that $T$. muris infection is delayed following depletion of CD4 T cells [51], inhibition/down-regulation of TH2 cytokines [33,45] and increased TH1 polarization [52]. It is therefore likely that our observation of reduced helminth-specific $\mathrm{TH} 2$ responses in this co-infection model could, at least in part, explain the delay in $T$. muris expulsion, although induction of TH1 immune responses to $M$. bovis BCG following T. muris infection would also influence parasite expulsion. Interestingly, altering the infection sequence to elucidate the effect of a subsequent mycobacterial infection on an established helminth-induced $\mathrm{TH} 2$ immune response did not have any negative influence on mycobacterial or helminth clearance by the host. This is most likely to be due to the rapid clearance of the helminth infection and development of resistance to re-infection, or due to the presence of an established TH1 immune response for altering helminth clearance [53].

These modified pathogen-specific and non-specific immune responses following co-infection provide clear evidence that both pathogens have the ability to reciprocally modulate immune responses towards each other at their individual infection foci. More importantly, the down-regulation of overall immune responsiveness in the context of both infections suggests co-infectioninduced immune suppression as a possible mechanism. Several reports confirm that chronic immune activation during helminth infections could initiate immune suppression or anergy [22]. Here, we show significant increases in the frequency of systemic $\mathrm{CD} 4^{+} \mathrm{T}$ cells and effector T cells in MLN of co-infected animals, suggesting increased immune activation following co-infection. Although the presence of immune suppressive regulatory cell populations was investigated, no differences in the frequencies of Treg populations could be detected between infection groups in either of the BALB/C coinfection models. As Treg cells exert their suppressive function in a cytokine dependent manner and also interact with other T cells and APC directly, the implications of co-infection on regulatory immune mechanisms are not clear. Changes in IL-10, Foxp3 and TGF- $\beta$ gene expression reveal that the role of Tregs cannot be excluded. Our results could point towards a role for other immune regulatory cell populations, and current research efforts are focused towards the involvement of innate nuocytes and myeloid derived suppressor cells (MDSCs) [54,55].

\section{Conclusion}

In summary, the work presented here supports the hypothesis that co-infection by two unrelated and anatomically separated pathogens can reciprocally alter the host's immune response to either infection. Co-infection 
altered host pathology and the host's ability to expel invading helminth parasites; however the magnitude of the impact was dependent on the sequence of co-infection. These phenotypic changes were associated with alterations in organ-restricted $\mathrm{TH} 1 / \mathrm{TH} 2 /$ Treg immune balance, immune suppression and pathogen-specific and non-specific cytokine responses. It is likely that multiple mechanisms may operate concurrently and further research is needed to identify the critical factors involved, although our results strongly support a mechanism whereby chronic immune activation leads to hyporesponsiveness resulting in reduced pathogenic control during co-infection. These findings demonstrate the complexity of immune response regulation and systemic interaction between innate and adaptive immunity and thereby hightlights the need for greater understanding of the role of infection history on the evolution of host immunity.

\section{Additional file}

Additional file 1: Figure S1. Representative histological $H$ \& E stained lung sections captured at 10x magnification illustrating the differences in histopathology between T. muris/BCG co-infected, BCG-only infected, uninfected and T. muris - only infected BALB/C mice infected according to experimental design as shown in Figure 1B.

\section{Competing interests}

The authors declare that they have no competing interests.

\section{Authors' contributions}

Study concept \& design - GW, HJN. Acquisition of data - HJN, LK. Statistical analysis - HJN, NDP. Analysis and interpretation of data - GW, HJN, NDP. Drafting of the manuscript - HJN, NDP. Critical revisions to the manuscript GW, AGL, NDP, PVH. Obtained Funding - GW, HJN. Study Supervision - GW All authors read and approved the final manuscript.

\section{Authors' information}

Hendrik J Nel and Nelita du Plessis co-first author.

\section{Acknowledgements}

This work was supported by the South African National Research Foundation and the South African Medical Research Council (MRC) through financial contributions to this project. We thank N. Brown for her technical assistance.

\section{Author details}

${ }^{1}$ Division of Molecular Biology and Human Genetics, MRC Centre for Molecular and Cellular Biology, NRF/DST Centre of Excellence in Biomedical TB Research, Faculty Medicine and Health Sciences, Stellenbosch University, Tygerberg, South Africa. ${ }^{2}$ University of Queensland Diamantina Institute, Brisbane, QLD, Australia.

Received: 11 July 2013 Accepted: 10 January 2014

Published: 17 January 2014

\section{References}

1. Bellamy R: Genetic susceptibility to tuberculosis. Clin Chest Med 2005 26:233-246. vi.

2. Hanekom M, van Pittius NC G, McEvoy C, Victor TC, Van Helden PD, Warren RM: Mycobacterium tuberculosis Beijing genotype: a template for success. Tuberculosis 2011, 91:510-523.

3. Schluger NW, Rom WN: The host immune response to tuberculosis. Am J Respir Crit Care Med 1998, 157:679-691.

4. WHO | The world health report 1999 - making a difference. http://www. who.int/whr/1999/en/index.html.
5. Elias D, Mengistu G, Akuffo H, Britton S: Are intestinal helminths risk factors for developing active tuberculosis? Trop Med Int Health 2006, 11:551-558

6. Hotez PJ, Molyneux DH, Fenwick A, Ottesen E, Ehrlich Sachs S, Sachs JD: Incorporating a rapid-impact package for neglected tropical diseases with programs for HIV/AIDS, tuberculosis, and malaria. PLoS Med 2006, 3:e102.

7. Adams JF, Schölvinck EH, Gie RP, Potter PC, Beyers N, Beyers AD: Decline in total serum lgE after treatment for tuberculosis. Lancet 1999, 353:2030-2033.

8. Flynn JL, Chan J: Immunology of tuberculosis. Annu Rev Immunol 2001, 19:93-129.

9. Dixon H, Blanchard C, Deschoolmeester ML, Yuill NC, Christie JW, Rothenberg ME, Else KJ: The role of Th2 cytokines, chemokines and parasite products in eosinophil recruitment to the gastrointestinal mucosa during helminth infection. Eur J Immunol 2006, 36:1753-1763.

10. Yazdanbakhsh M, van den Biggelaar A, Maizels RM: Th2 responses without atopy: immunoregulation in chronic helminth infections and reduced allergic disease. Trends Immunol 2001, 22:372-377.

11. Maizels RM, Balic A, Gomez-Escobar N, Nair M, Taylor MD, Allen JE: Helminth parasites-masters of regulation. Immunol Rev 2004, 201:89-116.

12. McKee AS, Pearce EJ: CD25 + CD4+ Cells contribute to Th2 polarization during helminth infection by suppressing Th1 response development. $\mathrm{J}$ Immunol 2004, 173:1224-1231.

13. Hesse M, Piccirillo CA, Belkaid Y, Prufer J, Mentink-Kane M, Leusink M, Cheever AW, Shevach EM, Wynn TA: The pathogenesis of schistosomiasis is controlled by cooperating IL-10-producing innate effector and regulatory T cells. J Immuno/ 2004, 172:3157-3166.

14. Borkow G, Weisman Z, Leng Q, Stein M, Kalinkovich A, Wolday D, Bentwich Z: Helminths, human immunodeficiency virus and tuberculosis. Scand J Infect Dis 2001, 33:568-571.

15. Bentwich Z, Kalinkovich A, Weisman Z, Borkow G, Beyers N, Beyers AD: Can eradication of helminthic infections change the face of AIDS and tuberculosis? Immunol Today 1999, 20:485-487.

16. Resende Co T, Hirsch CS, Toossi Z, Dietze R, Ribeiro-Rodrigues R: Intestinal helminth co-infection has a negative impact on both anti-mycobacterium tuberculosis immunity and clinical response to tuberculosis therapy. Clin Exp Immunol 2007, 147:45-52.

17. Babu S, Bhat SQ, Kumar NP, Jayantasri S, Rukmani S, Kumaran P, Gopi PG, Kolappan C, Kumaraswami V, Nutman TB: Human type 1 and 17 responses in latent tuberculosis are modulated by coincident filarial infection through cytotoxic T lymphocyte antigen-4 and programmed death-1. J Infect Dis 2009, 200:288-298.

18. Brown M, Mawa PA, Joseph S, Bukusuba J, Watera C, Whitworth JAG, Dunne DW, Elliott AM: Treatment of schistosoma mansoni infection increases helminth-specific type 2 cytokine responses and HIV-1 loads in coinfected Ugandan adults. J Infect Dis 2005, 191:1648-1657.

19. Elias D, Britton S, Kassu A, Akuffo H: Chronic helminth infections may negatively influence immunity against tuberculosis and other diseases of public health importance. Expert Rev Anti-Infect Ther 2007, 5:475-484.

20. Stewart GR, Boussinesq M, Coulson T, Elson L, Nutman T, Bradley JE: Onchocerciasis modulates the immune response to mycobacterial antigens. Clin Exp Immunol 1999, 117:517-523.

21. Elias D, Wolday D, Akuffo H, Petros B, Bronner U, Britton S: Effect of deworming on human $T$ cell responses to mycobacterial antigens in helminth-exposed individuals before and after bacille calmette-guérin (BCG) vaccination. Clin Exp Immunol 2001, 123:219-225.

22. Borkow $G$, Bentwich $Z$ : Chronic immune activation associated with chronic helminthic and human immunodeficiency virus infections: role of hyporesponsiveness and anergy. Clin Microbiol Rev 2004, 17:1012-1030

23. Malhotra I, Mungai P, Wamachi A, Kioko J, Ouma JH, Kazura JW, King CL: Helminth- and bacillus calmette-guérin-induced immunity in children sensitized in utero to filariasis and schistosomiasis. J Immunol 1999, 162:6843-6848.

24. Potian JA, Rafi W, Bhatt K, McBride A, Gause WC, Salgame P: Preexisting helminth infection induces inhibition of innate pulmonary anti-tuberculosis defense by engaging the IL-4 receptor pathway. J Exp Med 2011, 208:1863-1874.

25. Fulton SA, Martin TD, Redline RW, Henry Boom W: Pulmonary immune responses during primary mycobacterium bovis- calmette-guerin bacillus infection in C57BI/6 mice. Am J Respir Cell Mol Biol 2000, 22:333-343. 
26. Klementowicz JE, Travis MA, Grencis RK: Trichuris muris: a model of gastrointestinal parasite infection. Semin Immunopathol 2012, 34:815-828.

27. Wakelin D: Acquired immunity to trichuris muris in the albino laboratory mouse. Parasitology 1967, 57:515-524.

28. Else KJ, Wakelin D, Roach TI: Host predisposition to trichuriasis: the mouse-T. muris model. Parasitology 1989, 98(Pt 2):275-282.

29. Arocho A, Chen B, Ladanyi M, Pan Q: Validation of the 2-DeltaDeltaCt calculation as an alternate method of data analysis for quantitative PCR of BCR-ABL P210 transcripts. Diagn Mol Pathol 2006, 15:56-61.

30. Wang $X$, Seed B: A PCR primer bank for quantitative gene expression analysis. Nucleic Acids Res 2003, 31:e154

31. Aubin $E$, Lemieux R, Bazin R: Absence of cytokine modulation following therapeutic infusion of intravenous immunoglobulin or anti-red blood cell antibodies in a mouse model of immune thrombocytopenic purpura. Br J Haematol 2007, 136:837-843.

32. Hamelin M-Ė, Yim K, Kuhn KH, Cragin RP, Boukhvalova M, Blanco JCG, Prince GA, Boivin G: Pathogenesis of human metapneumovirus lung infection in BALB/c mice and cotton rats. J Virol 2005, 79:8894-8903.

33. Bancroft AJ, Artis D, Donaldson DD, Sypek JP, Grencis RK: Gastrointestinal nematode expulsion in IL-4 knockout mice is IL-13 dependent. Eur J Immunol 2000, 30:2083-2091.

34. Lamb TJ, Graham AL, Le Goff L, Allen JE: Co-infected C57BL/6 mice mount appropriately polarized and compartmentalized cytokine responses to litomosoides sigmodontis and leishmania major but disease progression is altered. Parasite Immunol 2005, 27:317-324.

35. Sangaré $L R$, Herrin BR, Herrin BR, John-Stewart G, Walson JL: Species-specific treatment effects of helminth/HIV-1 co-infection: a systematic review and meta-analysis. Parasitology 2011, 138:1546-1558

36. Erb KJ, Trujillo C, Fugate M, Moll H: Infection with the helminth nippostrongylus brasiliensis does not interfere with efficient elimination of mycobacterium bovis BCG from the lungs of mice. Clin Diagn Lab Immunol 2002, 9:727-730.

37. Frantz FG, Rosada RS, Turato WM, Peres CM, Coelho-Castelo AAM, Ramos SG, Aronoff DM, Silva CL, Faccioli LH: The immune response to toxocariasis does not modify susceptibility to mycobacterium tuberculosis infection in BALB/C mice. Am J Trop Med Hyg 2007, 77:691-698.

38. Elias D, Akuffo $H$, Thors C, Pawlowski A, Britton S: Low dose chronic Schistosoma mansoni infection increases susceptibility to mycobacterium bovis BCG infection in mice. Clin Exp Immunol 2005, 139:398-404.

39. Artis D, Potten CS, Else KJ, Finkelman FD, Grencis RK: Trichuris muris: host intestinal epithelial cell hyperproliferation during chronic infection is regulated by interferon- $\gamma$. Exp Parasitol 1999, 92:144-153.

40. Cliffe LJ, Potten CS, Booth CE, Grencis RK: An increase in epithelial cell apoptosis is associated with chronic intestinal nematode infection. Infect Immun 2007, 75:1556-1564.

41. Carmo AM, Vicentini MA, Dias AT, Alves LL, Alves CCS, Brandi JS, De Paula ML, Fernandes A, Barsante MM, Souza MA, Teixeira HC, Negrão-Corrêa D, Ferreira AP: Increased susceptibility to strongyloides venezuelensis in mice due to mycobacterium bovis co-infection which modulates production of Th2 cytokines. Parasitology 2009, 136:1357-1365.

42. Jenkins SN, Behnke JM: Impairment of primary expulsion of Trichuris muris in mice concurrently infected with nematospiroides dubius. Parasitology 1977, 75:71-78

43. Legesse M, Erko B, Balcha F: Increased parasitaemia and delayed parasite clearance in Schistosoma mansoni and plasmodium berghei co-infected mice. Acta Trop 2004, 91:161-166.

44. Phillips RS, Selby GR, Wakelin D: The effect of plasmodum berghei and trypanosoma brucei infections on the immune expulsion of the nematode Trichuris muris from mice. Int J Parasitol 1974, 4:409-415.

45. Cliffe $\amalg$, Humphreys NE, Lane TE, Potten CS, Booth C, Grencis RK: Accelerated intestinal epithelial cell turnover: a new mechanism of parasite expulsion. Science 2005, 308:1463-1465.

46. Khan Wl, Abe T, Ishikawa N, Nawa Y, Yoshimura K: Reduced amount of intestinal mucus by treatment with anti-CD4 antibody interferes with the spontaneous cure of Nippostrongylus brasiliensis-infection in mice. Parasite Immunol 1995, 17:485-491.

47. Else KJ, Hültner L, Grencis RK: Cellular immune responses to the murine nematode parasite Trichuris muris: II differential induction of TH-cell subsets in resistant versus susceptible mice. Immunology 1992, 75:232-237
48. Else KJ, Grencis RK: Antibody-independent effector mechanisms in resistance to the intestinal nematode parasite Trichuris muris. Infect Immun 1996, 64:2950-2954.

49. Wakelin D, Selby GR: Immune expulsion of Trichuris muris from resistant mice: suppression by irradiation and restoration by transfer of lymphoid cells. Parasitology 1976, 72:41-50.

50. Lee TD, Wakelin D, Grencis RK: Cellular mechanisms of immunity to the nematode Trichuris muris. Int J Parasitol 1983, 13:349-353.

51. Koyama K, Tamauchi H, Ito $Y$ : The role of CD4+ and CD8+ T cells in protective immunity to the murine nematode parasite Trichuris muris. Parasite Immunol 1995, 17:161-165.

52. Else KJ, Entwistle GM, Grencis RK: Correlations between worm burden and markers of Th1 and Th2 cell subset induction in an inbred strain of mouse infected with Trichuris muris. Parasite Immunol 1993, 15:595-600.

53. Bancroft AJ, Else KJ, Humphreys NE, Grencis RK: The effect of challenge and trickle Trichuris muris infections on the polarisation of the immune response. Int J Parasitol 2001, 31:1627-1637.

54. Nagaraj S, Collazo M, Corzo CA, Youn J-I, Ortiz M, Quiceno D, Gabrilovich DI: Regulatory myeloid suppressor cells in health and disease. Cancer Res 2009, 69:7503-7506

55. Neill DR, Wong SH, Bellosi A, Flynn RJ, Daly M, Langford TKA, Bucks C, Kane CM, Fallon PG, Pannell $R$, Jolin HE, McKenzie ANJ: Nuocytes represent a new innate effector leukocyte that mediates type-2 immunity. Nature 2010, 464:1367-1370.

doi:10.1186/1471-2180-14-9

Cite this article as: Nel et al:: Mycobacterium bovis BCG infection severely delays Trichuris muris expulsion and co-infection suppresses immune responsiveness to both pathogens. BMC Microbiology 2014 14:9.

\section{Submit your next manuscript to BioMed Central and take full advantage of:}

- Convenient online submission

- Thorough peer review

- No space constraints or color figure charges

- Immediate publication on acceptance

- Inclusion in PubMed, CAS, Scopus and Google Scholar

- Research which is freely available for redistribution

Submit your manuscript at www.biomedcentral.com/submit
C Biomed Central 